Research Article

\title{
Kinematics Study of Depth Jump on Male Triple Jumpers with Slope Run-Up
}

\author{
Youbao Ma 10 \\ Department of PE and Military Training, Zhejiang University of Technology, Hangzhou 310023, Zhejiang, China \\ Correspondence should be addressed to Youbao Ma; mayoubao@zjut.edu.cn
}

Received 14 July 2021; Revised 31 August 2021; Accepted 28 September 2021; Published 20 October 2021

Academic Editor: Sang-Bing Tsai

Copyright (c) 2021 Youbao Ma. This is an open access article distributed under the Creative Commons Attribution License, which permits unrestricted use, distribution, and reproduction in any medium, provided the original work is properly cited.

\begin{abstract}
Objective. To explore the training methods of developing the special strength of Chinese male triple jumpers through the experimental study of nine male triple-jumpers who performed step takeoff and depth long jump on the force platform. Methods. Through the combination of high-velocity shooting and a three-dimensional force measuring platform, the data of the athletes' full run-up and depth jump landing, horizontal velocity, vertical velocity, takeoff time, landing angle, takeoff angle, and strength were obtained. In this study, the kinematics characteristics of four slopes with different slopes of $25 \mathrm{~cm}$ and $35 \mathrm{~cm}$ were studied. Kinematics' data was measured by a high-velocity video camera. Results. The vertical velocity of the $35 \mathrm{~cm}$ platform is obviously less than that of the $25 \mathrm{~cm}$ platform $p<0.01$. The $25 \mathrm{~cm}$ platform is better at developing vertical, rapid takeoff ability, especially the fourth slope of this height, which is the fastest from horizontal velocity and vertical speed. All the depth jump practice ground angles are larger than the whole step jump, and the off-ground angle is smaller than the step jump, the takeoff time is larger than the step jump, which indicates that the athletes takeoff range is larger and the takeoff time is longer, and the results are in greater stimulation intensity of lower limb muscles. Conclusion. The $25 \mathrm{~cm}$ high platform depth jump exercise has achieved a better takeoff effect. Among the four slope depth jump exercises on this high platform, the 6.84 slope takeoff effect is the best.
\end{abstract}

\section{Introduction}

The triple jumper's vertical takeoff ability plays a very important role. Triple jumpers use various training methods to improve vertical takeoff ability. One of the most effective methods is depth jump exercises. The depth jump exercise is a typical plyometric exercise. Plyometric exercises make use of the stretch-shortening cycle by incorporating a rapid eccentric countermovement, quickly followed by a concentric movement $[1,2]$. These exercises are primarily used to promote improvements in power, enhance athletic performance, and prevent injury in athletes [Jensen and Ebben, 2007]. It is the rapid extension of muscles followed by the rapid contraction to produce greater strength and energy. The reason for its improved performance is believed to be caused by the rebound of the elastic structure of muscles and tendons and the increased reflex of muscle contraction, and this performance improvement is much better effective when the muscles are elongated and then contracted quickly.
In depth jump exercises, athletes are required to jump from a high place and then quickly take off and jump out of the best performance or highest height possible. Depth jump exercises can develop athletes' explosive power and enhance musculoskeletal function. However, scholars have not yet reached a consensus on the reasonable height of jump. Several research teams have conducted research on the height of jump practice. For example, Asmussen and Bonde Petersen (1974) used 14 men and 5 women as research subjects. After studying the vertical takeoff effect of three height jumps of $0.23 \mathrm{~m}, 0.40 \mathrm{~m}$, and $0.69 \mathrm{~m}$, it was found that the most effective height was $0.4 \mathrm{~m}$, which produced $0.408 \mathrm{~m}$ of vertical height. When the height increased to $0.69 \mathrm{~m}$, even though greater kinetic energy is generated, the athletes will use corresponding landing techniques to cushion the impact applied to them, thereby reducing the vertical takeoff effect. A study by Komi and Bosco (1978) shows that the best jump height for male athletes is 0.6 meters, while that for female athletes is $0.5 \mathrm{~m}$. The study of Bobbert el al. (1987a) shows 
that if the depth jumpers are allowed to land on the ground to limit their excessive cushioning and shorten the takeoff time, there is no significant difference in the height of the center of gravity rebound in the $0.2 \mathrm{~m}, 0.4 \mathrm{~m}$, and $0.6 \mathrm{~m}$ jump exercises. The athlete's ground reaction force increases with the increase of height, and the force value reaches the average value of $4496 \mathrm{~N}$ in the $0.6 \mathrm{~m}$ depth jump practice. ADRIAN LEES and EMAD FAHMI (1994) studied the jump depths of $0.12 \mathrm{~m}, 0.24 \mathrm{~m}, 0.36 \mathrm{~m}, 0.46 \mathrm{~m}, 0.58 \mathrm{~m}$, and $0.68 \mathrm{~m}$, and the results showed that the best performance in all measured parameters was for the drop height of $012 \mathrm{~m}$, including net height rise of the CG, maximum vertical force, maximum vertical velocity, and peak instantaneous power output derived from the product of force and velocity [3].

There are few studies on the approach run depth jump exercises for the triple jump. There is no research on the use of slope approach for depth jump special strength training for triple jumpers. According to the research by Sam J. Allen, M.R. (Fred) Yeadon, Mark A. King, etc., the strength of triple jumpers has a great influence on athlete performance. If an athlete with a score of $14.05 \mathrm{~m}$ increases their lower limb strength by $30 \%$, their performance can be increased to $16.20 \mathrm{~m}$, and if they increase their approach velocity by $30 \%$, their performance can only increase to $15.12 \mathrm{~m}$. In addition, as the strength increases, the ratio of triple jumps will change significantly. The greater the strength, the greater the proportion of stride jumps. If the velocity increases, but the strength does not increase, the proportion of stride jumps will decrease [5]. Therefore, improving the athlete's takeoff strength is extremely important for improving the athlete's performance and stride performance.

This platform can adjust the heights and slopes freely (Figure 1), and the adjustment of the slope angle is beneficial to the athletes to accelerate the approach and take off. Considering the case when an athlete performs depth jump exercises on a sloped high platform, the approach and takeoff of the athletes may change as the angle of the high platform changes. Therefore, we speculated that there must be a more suitable height and slope of the high platform, so that the depth jump and long jump exercises are more in line with the requirements of the special technical movements of the triple jump.

\section{Research Objects and Methods}

2.1. Research Objects. Nine male triple jumpers were recruited from Shanghai Institute of Physical Education: age $21.1 \pm 1$ years old, score $13.71 \pm 0.84 \mathrm{~m}$, average height $176.1 \pm 3.18 \mathrm{~cm}$, and average weight $69.2 \pm 6.29 \mathrm{~kg}$.

2.2. Research Methods. Biomechanical testing method (Figure 2): a Japanese JVC9800 video recorder was used to shoot the whole process of the triple long jump at a shooting velocity of 100 frames per second, and the main optical axis was aligned with the center of the force plate. The image was analyzed using the Myomotion system, and the shooting location was the indoor track and field stadium of the Shanghai University of Sport. The height of the front end was
$25 \mathrm{~cm}$ and $35 \mathrm{~cm}$, and the back end height was $0 \mathrm{~cm}, 20 \mathrm{~cm}$, $40 \mathrm{~cm}$, and $60 \mathrm{~cm}$ higher than the front end height, respectively. Its approach slopes were 0 degrees, 2.29 degrees, 4.58 degrees, and 6.84 degrees, which are called the first slope, the second slope, the third slope, and the fourth slope, respectively. Use 1-1, 1-2, 1-3, and 1-4 to represent the four slope exercises with a height of $25 \mathrm{~cm}$. 2-1, 2-2, 2-3, and 2-4 represent four slope exercises with a height of $35 \mathrm{~cm}$. The distance between the high platform and the force plate is $3.4 \pm 0.4 \mathrm{~m}$. The high-velocity video and the force plate were used to obtain relevant data synchronously, and then the kinematics was compared with the athlete's own full run-up stride. Athletes must try their best to run up and take off during the trial jump. Each person would perform 3 trial jumps, and finally we used the data with the best takeoff performance.

Mathematical statistics: we use IBM spss23 multifactor analysis of variance and paired $T$ test for hypothesis testing.

\section{Results and Analysis}

3.1. Analysis of Touchdown Velocity. Velocity is an important factor that affects the performance of the triple jump. According to the parabolic motion equation, the flight distance of each jump depends on the vertical velocity, horizontal velocity, and height of the body's center of gravity when taking off, and the height of the body's center of gravity when landing. The horizontal velocity and vertical velocity of step jump will directly affect the distance of stride jump, while the horizontal velocity and vertical velocity of depth jump will affect the strength training effect of depth jump. The greater the velocity, the greater the impact force and impulse when landing.

The approach of using a high platform slope is to quickly increase the approach velocity due to the downward movement of the center of gravity of the downhill running. At the same time, the horizontal component force generated by the takeoff on the slope is greatly increased, thereby increasing the horizontal velocity during the takeoff. Jumping from a high platform increases the height of the center of gravity. According to the free landing formula $v=g t$, the vertical touchdown velocity is proportional to the landing time. The higher the height, the longer the landing time, thus increasing the vertical velocity and improving the impulse when landing, thereby increasing the training intensity.

Table 1 illustrates the average value of the horizontal and vertical velocity at landing. Table 2 is a paired $T$ test for the horizontal velocity of the four slopes of the two high platforms. The study shows that there is no significant difference in the horizontal velocity of the depth jump at the first three slopes and two heights. The horizontal velocity at two heights of the first slope $(p=0.44)$, the second slope $(p=0.638)$, and the third slope $(p=0.772)$ and the horizontal velocity height of the fourth slope are significantly different $(p=0.001)$. Through the comparison of the average values, the horizontal velocity of the $25 \mathrm{~cm}$ high platform is obviously higher than the $35 \mathrm{~cm}$ high platform at the fourth slope, and the horizontal velocity and vertical velocity of the $35 \mathrm{~cm}$ fourth slope are lower than the first and 


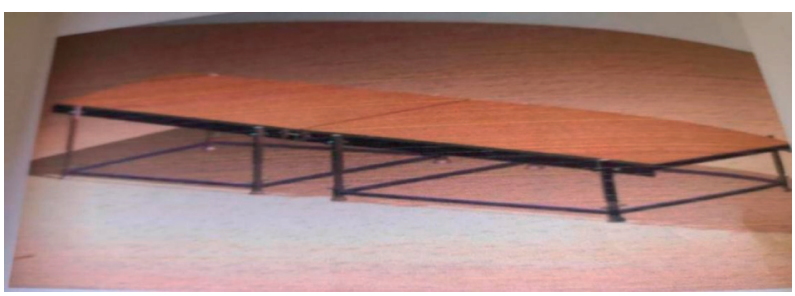

Figure 1: Self-made lifting platform diagram.

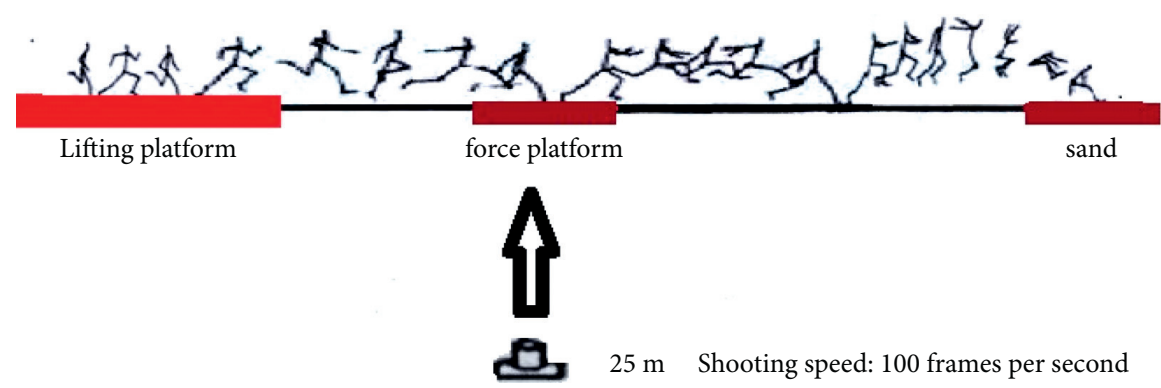

Figure 2: Schematic diagram of biomechanical testing [5].

second slopes (Table 1).Li Yanxi is a famous triple jumper in China, Asian record holder, whose landing horizontal and vertical velocity of his stride are shown in Table 1 when he jumped $17.09 \mathrm{~m}$ in 2004 . He has greater horizontal velocity and slower vertical velocity than the experimenters.

According to formula $V(V)=g t$, vertical landing velocity is proportional to time; this means that the subjects had longer flight time on hop, and they jumped higher than $\mathrm{Li}$ Yanxi on hop. The target of an efficient takeoff should be a compromise between a minimum loss of horizontal velocity and a good gain in vertical velocity. But the subjects may have large horizontal velocity loss.

From the perspective of the average vertical velocity, the $35 \mathrm{~cm}$ high platform is significantly higher than the $25 \mathrm{~cm}$ high platform, and the training vertical velocity of each high platform is significantly greater than the step jump, of which 2-1 and 2-2 are the largest (Table 1). After the $T$ test, when the slopes are the same, the vertical velocities of the two high platforms are significantly different $(p<0.01)$ Table 3 .

Table 3 shows that the difference of vertical velocity between the two platforms is significant. Table 1 shows that the vertical velocity of landing for $35 \mathrm{~cm}$ jump is faster than that for $25 \mathrm{~cm}$ jump. This demonstrates that, regardless of the slope, as long as the height of the high platform increases to the $35 \mathrm{~cm}$ platform, the vertical velocity of landing will increase significantly. It can be obtained by formula $P=M V$, ( $P$ is momentum, $M$ is the mass of the person, and $V$ is the velocity), so the vertical momentum will be greater. According to the theorem of momentum,

$$
F \Delta t=\sum m i^{\prime} v i^{\prime}-\sum m i v i=\sum p i^{\prime}-\sum p i .
$$

When $p$ increases, $F t$ increases, so $F$ increases, or $t$ increases too. When $F$ increases, it increases the intensity of stimulation to lower limb muscles, which helps improve specific muscle strength.
3.2. Analysis of Instantaneous Velocity off the Ground and Velocity Utilization. Sam J. Allen (2012) research showed that the loss in horizontal velocity during each contact phase was strongly related to the vertical takeoff velocity $(R 21 / 40.83)$ in that phase rather than the overall gain in vertical velocity as found in previous studies. Maximum overall distances were achieved with step phases, which were $30 \%$ of the total distance of the triple jump confirming the results of experimental studies on elite triple jumpers [4].

When the famous Chinese Athlete Li Yanxi jumped $17.09 \mathrm{~m}$ in 2004 , the horizontal velocity and the vertical velocity of step jump off the ground were $7.8 \mathrm{~m} / \mathrm{s}$ and $1.78 \mathrm{~m} /$ $\mathrm{s}$, respectively. The $\mathrm{Hu}$ and the $\mathrm{Vu}$ of Yanxi $\mathrm{Li}$ are $93.98 \%$ and $100 \%$. The horizontal velocity off the ground in this study was $5.788 \mathrm{~m} / \mathrm{s}$, and the vertical velocity was $1.696 \mathrm{~m} / \mathrm{s}$. The vertical velocity off the ground for the $25 \mathrm{~cm}$ high platform depth jump practice is not only greater than that of the stride jump, but also greater than Li Yanxi's vertical jump speed (Table 4).

A study demonstrates that phase ratio significantly affects the actual distance of the triple jump, and approach run velocity is another factor that affects the performance of the triple jump [4]. Table 4 shows that 1-3 and 1-4 exercises vertical velocity conversion coefficient is higher than that of other depth jumps, and 2-1 horizontal velocity conversion coefficient is the highest, and the vertical velocity utilization rate is only $47.01 \%$, the lowest in all exercises, so 1-3 and 1-4 exercises have better effect.

The landing and off ground horizontal velocities of various depth jump exercises are all lower than the stride jump, and the utilization rate of the horizontal velocity is greater than the stride jump. The vertical landing velocity of the depth jump practices is significantly higher than that of the stride jump, but there are obvious differences in the vertical velocity. The $25 \mathrm{~cm}$ platform is greater than the stride jump, and the second high platform is 
TABLE 1: The average value of the horizontal and vertical velocity at landing $(\mathrm{m} / \mathrm{s})$.

\begin{tabular}{lcccccccccc}
\hline High platform changes & Li Yanxi & Whole course & $1-1$ & $1-2$ & $1-3$ & $1-4$ & $2-1$ & $2-2$ & $2-3$ & $2-4$ \\
\hline Horizontal & 8.3 & 6.8 & 5.74 & 5.34 & 5.43 & 5.93 & 5.38 & 5.42 & 5.34 & 5.22 \\
Standard deviation & & 0.653 & 0.885 & 0.459 & 0.426 & 0.325 & 0.445 & 0.579 & 0.418 & 0.526 \\
Vertical & \multirow{2}{*}{1.78} & 1.91 & 2.697 & 2.493 & 2.502 & 2.423 & 2.801 & 2.873 & 2.672 & 2.582 \\
Standard deviation & & 0.532 & 0.381 & 0.309 & 0.210 & 0.376 & 0.413 & 0.311 & 0.228 & 0.317 \\
\hline
\end{tabular}

TABLE 2: Paired $T$ test of horizontal velocity on the same slope.

\begin{tabular}{lcccccc}
\hline & & Average value & Standard deviation & $\mathrm{t}$ & Degree of freedom & Significance (two-tailed) \\
\hline Pairing 1 & $1-1$ and 2-1 & -0.11 & 0.70 & -0.784 & 26 & 0.440 \\
Pairing 1 & $1-2$ and 2-2 & 0.059 & 0.64 & 0.477 & 26 & 0.638 \\
Pairing 1 & $1-3$ and 2-3 & 0.043 & 0.62 & 0.360 & 26 & 0.722 \\
Pairing 1 & $1-4$ and 2-4 & 0.55 & 0.80 & 3.592 & 26 & 0.001 \\
\hline
\end{tabular}

TABLE 3: Paired $T$ test of vertical velocity at the same slope and different height.

\begin{tabular}{|c|c|c|c|c|c|c|c|c|c|}
\hline \multicolumn{10}{|c|}{ Paired sample test } \\
\hline \multicolumn{10}{|c|}{ Paired difference } \\
\hline & & \multirow{2}{*}{ Average value } & \multirow{2}{*}{$\begin{array}{l}\text { Standard } \\
\text { deviation }\end{array}$} & \multirow{2}{*}{ Standard error mean } & \multicolumn{2}{|c|}{$\begin{array}{c}\text { Difference } 95 \% \text { confidence } \\
\text { interval }\end{array}$} & \multirow[t]{2}{*}{$t$} & \multirow[t]{2}{*}{$\begin{array}{l}\text { Degree of } \\
\text { freedom }\end{array}$} & \multirow[t]{2}{*}{$\begin{array}{l}\text { Significance } \\
\text { (two-tailed) }\end{array}$} \\
\hline & & & & & Lower limit & Higher limit & & & \\
\hline Pairing & $1-1$ and $2-1$ & 0.30593 & 0.47219 & 0.09087 & 0.11913 & 0.49272 & 3.367 & 26 & 0.002 \\
\hline Pairing & $1-2$ and $2-2$ & 0.46963 & 0.54576 & 0.10503 & 0.25373 & 0.68553 & 4.471 & 26 & $<0.001$ \\
\hline Pairing & $1-3$ and $2-3$ & 0.60363 & 0.40153 & 0.07727 & 0.44479 & 0.76247 & 7.811 & 26 & $<0.001$ \\
\hline Pairing & $1-4$ and $2-4$ & 0.27000 & 0.43030 & 0.08281 & 0.09978 & 0.44022 & 3.260 & 26 & 0.003 \\
\hline
\end{tabular}

TABLe 4: Horizontal $(\mathrm{H})$ velocity, vertical $(\mathrm{V})$ velocity, and velocity utilization rate off the ground $(\mathrm{m} / \mathrm{s})$.

\begin{tabular}{lccccccccc}
\hline High platform & Step & $1-1$ & $1-2$ & $1-3$ & $1-4$ & $2-1$ & $2-2$ & $2-3$ & $2-4$ \\
\hline$H$ velocity & 5.788 & 5.13 & 4.99 & 4.91 & 5.18 & 5.17 & 4.763 & 4.712 & 4.724 \\
$H$ u \% & 85.12 & 89.37 & 93.45 & 90.42 & 87.35 & 95.92 & 89.87 & 87.53 & 90.19 \\
$V$ velocity & 1.696 & 1.759 & 1.867 & 1.935 & 1.957 & 1.368 & 1.516 & 1.404 & 1.432 \\
$V u \%$ & 88.79 & 66.96 & 80.31 & 83.38 & 80.77 & 47.01 & 54.26 & 48.02 & 53.17 \\
\hline
\end{tabular}

significantly less than the stride jump. After testing, there is a significant difference in vertical takeoff velocity between the two heights $(p<0.01)$, which shows that the first high platform produces a larger upward momentum, which has a better effect on the improvement of the athlete's vertical takeoff ability (Table 5).

Relevant studies have shown that the actual distance obtained using the optimal phase ratio significantly increased as the horizontal velocity at the landing of the last step of approach run increased $(p=0.001)$, and the corresponding downward vertical velocity decreased $(p=0.001)$; each step of the jump will lose a certain horizontal velocity, and the reduction of the horizontal velocity of stepping is in the range of $7 \%$ to $15 \%$. The Chinese scholar Yu-Jingping found that the world's outstanding male triple jump athletes have a loss rate of $10.5 \%$ in stride speed. The world record holder Edwards' horizontal velocity dropped by $11.6 \%$, and Wilman dropped by $11.8 \%$. [6] In this study, the horizontal velocity loss rate of the depth jump is between $12.65 \%$ and $6.55 \%$, which is significantly less than the $14.88 \%$ horizontal velocity loss rate of the stride jump. Therefore, the velocity utilization rate of depth jump exercises is in line with the world's high level requirements, and regular training can improve the velocity utilization rate of athletes.

Table 4 shows that the horizontal velocity utilization rate of various jump depths is significantly greater than the horizontal velocity utilization rate of the stride jump, while the vertical velocity utilization rate of the depth jump exercises is significantly less than that of the stride jump; there is no significant difference in the horizontal velocity utilization rate between the two heights. In the case of $25 \mathrm{~cm}$ platform, the vertical velocity utilization rate of the three depth jump exercises with slop is significantly higher than the 1-1 exercises without slope, and there are significant differences in height $(p=0.004<0.01)$. In the case of $35 \mathrm{~cm}$ platform, the vertical velocity utilization of 2-2, 2-3, and 2-4 exercises with slope is also higher than that of the 2-1 exercises without slope, but there is no significant difference $(p=0.13)$. This suggests that athletes practicing vertical takeoff at on the $35 \mathrm{~cm}$ platform produce a greater vertical strike force or impulse, which results in a greater loss of the athlete's vertical speed. 
TABLE 5: Vertical velocity off-ground inspection.

\begin{tabular}{|c|c|c|c|c|c|c|c|c|c|c|}
\hline \multicolumn{6}{|c|}{ Levine variance equivalence test } & \multicolumn{5}{|c|}{ Mean equality $t$-test } \\
\hline & & \multirow{2}{*}{$F$} & \multirow{2}{*}{ Significance } & \multirow{2}{*}{$\mathrm{t}$} & \multirow{2}{*}{$\begin{array}{l}\text { Degree of } \\
\text { freedom }\end{array}$} & \multirow{2}{*}{$\begin{array}{l}\text { Significance } \\
\text { (two-tailed) }\end{array}$} & \multirow{2}{*}{$\begin{array}{c}\text { Mean } \\
\text { difference }\end{array}$} & \multirow{2}{*}{$\begin{array}{l}\text { Standard } \\
\text { error } \\
\text { difference }\end{array}$} & \multicolumn{2}{|c|}{$\begin{array}{c}\text { Difference 95\% } \\
\text { confidence interval }\end{array}$} \\
\hline & & & & & & & & & $\begin{array}{l}\text { Lower } \\
\text { limit }\end{array}$ & $\begin{array}{c}\text { Higher } \\
\text { limit }\end{array}$ \\
\hline \multirow{2}{*}{$\begin{array}{l}\text { Vertical } \\
\text { speed }\end{array}$} & $\begin{array}{l}\text { Assumed equal } \\
\text { variance }\end{array}$ & 0.593 & 0.471 & 8.243 & 6 & $0.000<0.001$ & 0.4495000 & 0.0545306 & 0.3160685 & 0.5829315 \\
\hline & $\begin{array}{l}\text { Doesn't assume } \\
\text { equal variance }\end{array}$ & & & 8.243 & 5.405 & $0.000<0.001$ & 0.4495000 & 0.0545306 & 0.3124233 & 0.5865767 \\
\hline
\end{tabular}

3.3. Characteristics of Landing Angle and Takeoff Angles. Figure 3 shows the landing angle, takeoff angle, braking phase, and pushing phase. The landing angle refers to the angle of the line between the center of gravity and the foot and the horizontal direction at the instant of landing. Increasing the landing angle and reducing the horizontal braking force will help the body's center of gravity move forward quickly and increase the horizontal speed. However, to maintain high horizontal speed throughout the triple jump, the inclination of the braking angle of the average resultant force should be closer to $90^{\circ}$ [7]. However, the following is the increase in the moment of inertia, and it is easy for the athlete to lose balance.

When jumping at narrow takeoff angles, the jumper had a high horizontal speed, and so he could land with his feet far ahead of his body without the risk of falling backward after landing. When landing from a high wide takeoff angles, the jumper had a lower horizontal speed and so landed in a nearly upright position with the feet only slightly ahead of the body. Therefore, the landing height steadily increased, and the landing distance steadily decreased as the takeoff angle increased [8]. The horizontal velocity is slower on the practices of depth jump than the triple jump, and the moment of inertia is relatively small, so the takeoff and landing angle can be appropriately increased. James Hay proposed that the analysis of the athlete's technique from the landing angle and the takeoff angle shows that when the landing angle is larger, it is beneficial to shorten the horizontal braking time. When the takeoff angle is small, it is beneficial to increase the horizontal speed. Both can slow down the horizontal velocity in the takeoff phase and cause a certain level of velocity loss. At the same time, the vertical landing velocity also increases with the height of the high platform. When the landing vertical velocity is very high, an athlete has to increase the upward takeoff velocity and reduce the ground reaction forces by extending the takeoff time; this leads to an increase in horizontal velocity loss. So, excellent athletes actively swing the jumping leg backwards prelanding, greatly reducing the horizontal braking when landing, increasing the angle of landing, and increasing the vertical speed. The landing angle for depth jump exercises is significantly greater than the landing angle for full stride jumps; see Table 6.

It can be seen from Table 6 that the landing angle of athletes is larger, and the takeoff angle is smaller than that of Li Yanxi. It may be related to the slower velocity of athletes at this level, insufficient forward extension of the takeoff legs, and slow extension of the hip joints, which lead to the relatively forward center of gravity of the body. On the other

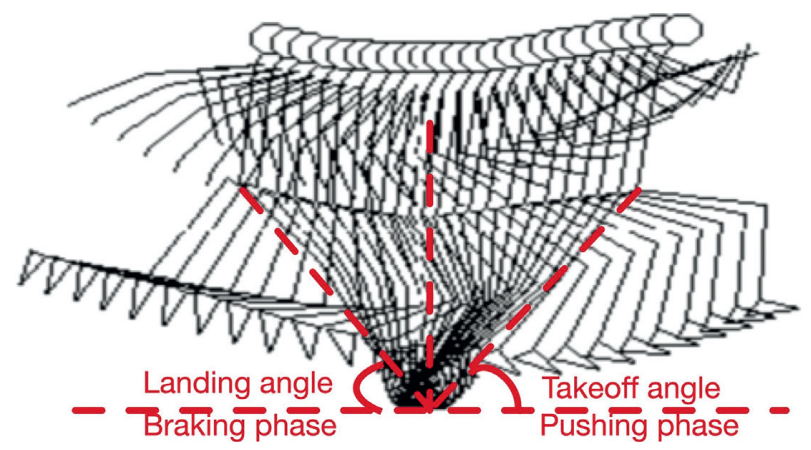

FIgURE 3: The schematic diagram on step jump of the triple jump.

hand, we believe that depth jump exercises increase the landing angle, reduce the braking horizontal impulse, and increase the vertical braking impulse, which have a positive effect on the development of athletes' vertical takeoff ability.

After $T$ test (Table 7), the landing angle of each high platform practice is significantly different from the stride jump $(p<0.01)$, and the $2-4$ landing angle is significantly different from other depth jump exercises $(p<0.01)$. However, the landing angles of 1-1 and 2-3 were significantly different from other exercises ( $p=0.038,0.012$ ), and there were no significant differences among other exercises $(p>0.05)$.

Different athletes have a big difference in the landing angle. In the 15th World Athletics Championships, the triple jump champion Taylor's landing angle was 66 degrees at $18.21 \mathrm{~m}$, and the off-ground angle was 63 degrees [9], while Li Yanxi was 70.3 degrees and 68.5 degrees at $17.59 \mathrm{~m}$ in 2009 [10]. If Taylor and Li Yanxi had the same ground contact force at landing and off the ground, Taylor horizontal braking force and driving force might be bigger than those of Li yanxi due to the different landing and takeoff angle.

From the perspective of the average values of takeoff angles, the fourth slope is larger, and the takeoff angles of all the depth jump are less than those of the stride jump. The larger the landing angle is, and the smaller the takeoff angle is, the more it reduces the forward resistance and increases the forward driving force. Therefore, we believe that depth jump practice is a better method of special strength training.

Why is takeoff angle significantly reduced in depth jump exercises? We think it may be because the vertical strike force is larger, and athletes need greater cushioning knee angles, so the body's center of gravity is lower during takeoff. This feature of depth jump exercises is conducive to maintaining the horizontal speed, which is why the 
TABLE 6: Mean value of landing and off-ground angles (degree).

\begin{tabular}{lcccccccccc}
\hline High platform & Liya Nxi & Step & $1-1$ & $1-2$ & $1-3$ & $1-4$ & $2-1$ & $2-2$ & $2-3$ & $2-4$ \\
\hline Landing angle & 70.3 & 70.61 & 73.39 & 74.64 & 74.49 & 74.71 & 74.07 & 73.74 & 75.02 & 72.67 \\
Leaving angle & 68.5 & 62.87 & 58.64 & 58.16 & 58.49 & 60.71 & 59.55 & 58.41 & 58.93 & 63.87 \\
\hline
\end{tabular}

TABle 7: Difference test of landing angle between depth jump exercise and step jump.

\begin{tabular}{cccccc}
\hline & Test value & $\mathrm{t}$ & Degree of freedom & Significance & Average difference \\
\hline \multirow{3}{*}{ Landing angle } & $70.61($ step) & 12.595 & 7 & $<0.001$ & 3.48375 \\
& $72.67(2-4)$ & 5.147 & 7 & 0.001 & 1.42375 \\
& $73.39(1-1)$ & 2.544 & 7 & 0.038 & 0.70375 \\
& $75.02(2-3)$ & -3.349 & 7 & 0.012 & -0.92625 \\
\hline
\end{tabular}

utilization of the horizontal velocity of the depth jump exercises is significantly greater than that of the step jump (see Table 4).

After testing, there is a significant difference taking off angle between the depth jump and the stride jump $(p=0.002)$, and the average value of other depth jump exercises and 2-4 exercises has a significant difference $(p=0.001)$. There was no significant difference among the other 7 depth jump exercises $(p>0.05)$, indicating that the 2-4 exercise has the smallest forward extension, which may not be conducive to the development of athletes' extension ability.

3.4. Analysis of the Maximum Buffer Knee Angle. The change of knee joint angle during takeoff stage buffering and kickextension conversion process is an important evaluation index of kinetic energy transmission. The maximum knee flexion range is 31 to 35 degrees in the stride jump. The smaller the change, the stronger the support strength [1]. Some literature pointed out [3] that, on elite male triple jumpers in China, the mean of the knee joint angle of the supporting leg at landing was 156.8 degrees, and the maximum buffer angle was $(122.16 \pm 6.70)$ degrees in step jump, and the maximum cushioning angle is $(130 \pm 3)$ degrees in the world excellent triple jumpers. In a jump that set a world record of $18.29 \mathrm{~m}$, Edwards stepped to the ground with a knee angle of 168.06 degrees and a maximum cushion knee angle of 133.21 degrees. In this study, the knee angle of the athletes in the stride jump is slightly larger than the average value of the excellent male triple jumpers in China and in the world. In all the depth jump exercises, only 1-4 has a smaller buffer knee angle than the stride jump; see Table 8 .

The smaller the maximum cushioning knee angle, the greater the cushioning amplitude. The cushioning knee angle is produced by muscle concessional work and is the result of muscle eccentric contraction. The larger the cushioning knee angle, the smaller the concessional work range. Many studies have shown that muscle regressive contraction is more effective than restrained contraction in improving the fast takeoff ability of practitioners. The larger buffer amplitude of 1-4 depth jump exercises is in line with the principle of large eccentric contraction and rapid concentric contraction of the muscles, resulting in a larger takeoff speed.
1-1, 2-1, and 2-2 exercises have a larger buffer knee angle, while other exercises have no significant difference with the fullcourse jump. The standard deviation of the maximum buffer knee angle for each depth jump exercise is much greater than that of the stride jump. It can be seen that the maximum buffer knee angle of athletes has a greater differentiation. When the athlete's lower limbs are hit hard, they often actively buffer and increase the buffer amplitude. Some athletes use strong support to counteract the impact of the lower limbs, and the athletes have endured huge impact. We suggest that athletes need to make greater active buffering at the beginning of training and increase the buffering range and leg drive range to avoid sports injuries caused by excessive impact. In this experiment, the maximum vertical ground reaction forces 10.94-17.6 times the weight of the athletes. Such a large impact indicates that some athletes should increase the cushioning amplitude of the knee joint. It is worth noting that the buffering amplitude of 1-4 exercises is larger, and the buffering knee angle is smaller than the stride jump, combined with the analysis of the larger horizontal and vertical velocity off the ground. We believe that this exercise has produced a greater takeoff effect, and athletes have increased their velocity off ground through greater cushioning and extension range. In addition, the maximum cushion knee angle of the 1-3 exercise is also smaller, which also produces a larger vertical velocity, but its horizontal velocity is smaller, which shows that this exercise increases the vertical velocity by reducing the horizontal velocity.

\subsection{The Characteristics of Braking Time and Pushing Time for} Stride Jump and Depth Jump Exercises. Studies showed that the stride time of the world's outstanding male triple jumpers varies from 0.14 to 0.17 seconds, with an average value of 0.15 seconds. Regarding Taylor's stride jump, the braking time has been maintained at 0.06 seconds, but the pushing time has been extended by 0.02 seconds. For Taylor, the appropriate extension of the pushing time increased the takeoff effect. [9]. In the Tenth National Games, the pushing time of Chinese male triple jumpers ranged from 0.06 seconds to 0.1 seconds, with an average of 0.078 seconds. The average time of the world's excellent athletes was 0.06 seconds. The test subject's time was 0.0856 seconds, which was longer than the average pushing time of the high-level world and Chinese triple jumpers, while the takeoff time was 


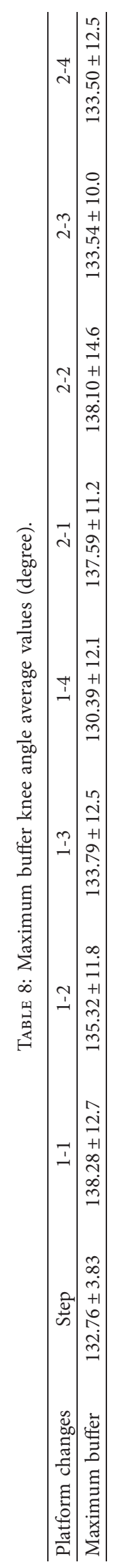


TABLE 9: Mean of braking time and pushing time (s).

\begin{tabular}{lccccccrrr}
\hline Platform changes & Step & $1-1$ & $1-2$ & $1-3$ & $1-4$ & $2-1$ & $2-2$ & $2-3$ & $2-4$ \\
\hline Braking time & 0.0930 & 0.1067 & 0.1163 & 0.1165 & 0.1167 & 0.1163 & 0.1219 & 0.1136 & 0.1215 \\
Pushing time & 0.0856 & 0.1056 & 0.1060 & 0.1035 & 0.1063 & 0.0946 & 0.0981 & 0.1236 & 0.1008 \\
Total time & 0.1786 & 0.2123 & 0.2223 & 0.2200 & 0.2230 & 0.2109 & 0.2200 & 0.2372 & 0.2223 \\
\hline
\end{tabular}

0.1786 seconds, which was higher than the world's elite athletes' average of 0.15 seconds.

The braking time and pushing time of depth jump exercises are longer than the step jump, and both have significant differences between the $25 \mathrm{~cm}$ and the $35 \mathrm{~cm}$ heights $(p \leq 0.001)$. The long buffer time is mainly due to the slower horizontal velocity of landing and the faster vertical speed, which results in slower forward movement, thereby prolonging the buffer time; The long driving time is due to the slow horizontal speed on the one hand and, on the other hand, to the huge momentum it takes when landing. It is quite difficult to quickly stretch under the action of overcoming the huge impact force, especially the insufficient horizontal stretch force, which leads to the increase of the pushing time, as shown in Table 9.

From the comparison of the braking time and the pushing time, only the pushing time of 2-3 is longer than the braking time, and the stretch time of the others is shorter. After paired $T$ test, there is a significant difference between the braking time and the pushing time $(p=0.026>0.05)$. Except 2-3, the pushing time of the second high platform is shorter than that of the first high platform. There are two reasons for the short pushing time: one is the fast takeoff speed, and the other is the small stretch range. Combined with the analysis of horizontal speed, vertical speed, buffer knee angle, landing angle, and takeoff angle, the longer pushing time of 2-3 exercise is mainly caused by slow velocity and large takeoff range.

There was no significant difference in the braking time between the two high platform depth jump exercises $(p=0.831>0.05)$, but the difference in the stretch time was significant $(p=0.046<0.05)$. The average values of the kicking time of the first high platform and the second high platform are 0.10535 seconds and 0.104275 seconds, respectively. Therefore, it can be considered that the second high platform has a shorter stepping time. If it were not for the larger stepping time caused by $2-3$, the difference between the two high platform steps would be more significant, and the second high platform would have a shorter time. The slow takeoff velocity indicates that the athlete's kicking and stretching are insufficient, and the kicking strength is insufficient. During training, the second high platform exercise should appropriately increase the buffering range, reduce the horizontal braking force, and increase the leg swing speed. In addition, there was no significant difference in the total takeoff time between the two high platforms $(p=0.612>0.05)$.

\section{Conclusion}

(1) The vertical velocity of the $25 \mathrm{~cm}$ platforms is obviously higher than that of the step jump and the $35 \mathrm{~cm}$ platforms; especially the takeoff horizontal and vertical velocity of the 1-4 platform are the largest. The landing angles of all depth jump are larger, takeoff angles except 2-4 are all smaller than those of the whole step jump, and there are significant differences, which indicates that the distance of horizontal braking of depth-jump athletes is small, and the distance of drive is large. Combined with the analysis of the maximum angle of the knee joint of the third and fourth slopes of the two platforms, the range of extension phase of the jump may be greater. Therefore, we believe that 1-3 and 1-4, especially 1-4, may have a better effect on improving takeoff ability of stretch-shortening cycle of athletes.

(2) The vertical landing velocity of the $35 \mathrm{~cm}$ platform and the knee angles buffer range are obviously larger than those of the step jump and the $25 \mathrm{~cm}$ platform, which indicates that the $35 \mathrm{~cm}$ platform athletes bear a large load, which leads to the slow speed of the athletes off the ground and the difficulty of taking off. In particular, the first and second slope exercises of the $35 \mathrm{~cm}$ platform produce a larger vertical landing speed. We believe that the $35 \mathrm{~cm}$ exercises can be used to develop the athletes' concession ability; especially 2-2 may have a better effect on improving the climb-down capability for the triple jumpers, but it is not suitable to develop the athletes' ability to stretchshortening cycle.

\section{Suggestion}

(1) Elevating platform can be used to develop the takeoff ability of athletes in other sports, including long jump and high jumpers. Coaches should refer to the athletes' ability to successfully complete the entire technical movement. When athletes have difficulty in taking off, they should lower the height and slope of the high platform.

(2) The depth jump exercises for triple jumpers stimulate the muscles and nervous system greatly, The ground reaction forces acting on the limbs during plyometric exercises are important to consider, as they place stresses on the body that may influence training-related neuromuscular adaptations and injury risk [1], so the training load should be less, generally 2-3 times a week, with 20-30 exercises each time. For high-level athletes, do not exceed 40 exercises each time. Coaches should pay attention to the athlete's takeoff velocity and final results. When the performance drops, they should stop the depth jump practice.

(3) It is recommended not to conduct such training two weeks before the competition. 


\section{Data Availability}

The data that support the findings of this study are available from the corresponding author upon reasonable request.

\section{Conflicts of Interest}

The authors declare that they have no known competing financial interests or personal relationships that could have appeared to influence the work reported in this paper.

\section{Acknowledgments}

This project was supported by Shanghai University of Sports.

\section{References}

[1] R. L. Jensen and W. P. Ebben, "Quantifying plyometric intensity via rate of force development, knee joint, and ground reaction forces," The Journal of Strength \& Conditioning Research, vol. 21, no. 3, pp. 763-767, 2007.

[2] J. N. Wilder, E. R. Riggins, R. A. Noble, C. M. Lelito, T. L. Widenhoefer, and T. G. Almonroeder, "The effects of drop vertical jump technique on landing and jumping kinetics and jump performance," Journal of Electromyography and Kinesiology, vol. 56, p. 102504, 2021.

[3] A. Lees and E. Fahmi, "Optimal drop height for polymeric training," Ergonomics, vol. 37, no. 1, pp. 141-148.

[4] S. J. Allen, M. A. King, and M. R. Yeadon, "Trade-offs between horizontal and vertical velocities during triple jumping and the effect on phase distances," Journal of Biomechanics, vol. 46, no. 5, pp. 979-983, 2013.

[5] S. J. Allen, M. R. Yeadon, and M. A. King, "The effect of increasing strength and approach velocity on triple jump performance," Journal of Biomechanics, vol. 49, no. 16, pp. 3796-3802, 2016.

[6] J.-P. Yu, "The analysis on technical characteristics of elite male triple jumpers at home and abroad," China Sport Science and Technology, vol. 39, no. 8, 2003.

[7] J. O. Perttunen, H Kyröläinen, P. V Komi, and A Heinonen, "Biomechanical loading in the triple jump," Journal of Sports Sciences, vol. 18, pp. 363-70, 2000.

[8] M. Wakai and N. P. Linthorne, "Optimum take-off angle in the standing long jump," Human Movement Science, vol. 24, no. 1, pp. 81-96, 2005.

[9] F. Zheng, T. Yuan, and A. Li, "Analysis on take-off techniques of Taylor 18.21 meters of the mens TripleJump final match in the 15th," IAAF World Championships China Sports Science and Technology, vol. 52, no. 6, pp. 99-106, 2016.

[10] J. Li, "Analysis of the first jump technique of record-breaking triple jumper Li Yanxi," Journal of Chengdu Institute of Physical Education, vol. 36, no. 10, pp. 41-60, 2010. 